\title{
Current Studies on a Working Model of CaM Kinase II in Hippocampal Long-Term Potentiation and Memory
}

\author{
Kohji Fukunaga and Eishichi Miyamoto \\ Department of Pharmacology, Kumamoto University School of Medicine, 2-2-1 Honjo, Kumamoto 860-0811, Japan \\ Received October 13, 1998
}

\begin{abstract}
There have been recent advances in understanding the molecular basis of the long-term potentiation (LTP) of synaptic transmission in the hippocampus. This review documents current views on mechanisms underlying LTP induction, from activation of the NMDA receptor to stimulation of $\mathrm{Ca}^{2+}$ /calmíodulin-dependent protein kinase II (CaM kinase II). We will focus in particular on recent findings of how CaM kinase II encodes the frequency of synaptic usage and serves as a molecular memory switch at the synapse. Furthermore, a role for CaM kinase II in spatial learning and memory is demonstrated by recent studies using transgenic mice.
\end{abstract}

Keywords: $\mathrm{Ca}^{2+} /$ calmodulin-dependent protein kinase II, Long-term potentiation, Long-term depression, Hippocampus, Spatial learning

CaM kinase II works as a $\mathrm{Ca}^{2+}$ pulse frequency detector $\mathrm{Ca}^{2+} /$ calmodulin-dependent protein kinase II (CaM kinase II) is concentrated in the brain and is enriched at synaptic structures, where it comprises $10-20 \%$ of total protein. The enzyme forms a homo-oligomeric structure composed of 8-12 subunits of the $\alpha$ - or $\beta$-isoform. An interesting feature of the enzyme is that autophosphorylation of Thr- 286 converts CaM kinase II from a $\mathrm{Ca}^{2+}$ dependent form to a $\mathrm{Ca}^{2+}$-independent form (autonomous kinase) $(1,2)$ (Fig. 1). Phosphorylation of Thr-286 in one subunit facilitates phosphorylation of Thr-286 in a second subunit, since Thr-286-phosphorylated subunits can phosphorylate an adjacent subunit in the presence of $\mathrm{Ca}^{2+} / \mathrm{CaM}(3,4)$. In addition, autophosphorylation of Thr-286 increases the dissociation constant for CaM from about $0.2 \mathrm{sec}$ in the unphosphorylated form to about $20 \mathrm{sec}$ in the phosphorylated form and results in trapping of CaM. The multimeric form of the kinase is required to generate its autonomous activities, since one subunit binding CaM can function as a "kinase" for a second proximal calmodulin-bound subunit serving as "substrate" (3). This reaction has a requirement for $\mathrm{Ca}^{2+}$ /CaM binding on both the "kinase" and the "substrate" subunits and therefore requires two molecules of CaM to generate the autonomous and trapped states (3) Thus, maximal activation of the autonomous kinase with more than $70 \% \mathrm{Ca}^{2+}$-independence is obtained by autophosphorylation at 1-2 mol phosphate/mol holoenzyme. High $\mathrm{Ca}^{2+}$ signaling leading to high levels of $\mathrm{CaM}$ occupancy would facilitate such an autonomous state. Like autophosphorylation of Thr-286, positive cooperativity is also evident in CaM binding to each subunit. Taken together, autophosphorylation of CaM kinase II can encode transient but large increases in postsynaptic $\mathrm{Ca}^{2+}$ mediated by NMDA receptors following high frequency stimulation inducing long-term potentiation (LTP). A high threshold of $\mathrm{Ca}^{2+}$ (more than $1 \mu \mathrm{M}$ ) is required to generate a positive cooperative state. Once $\mathrm{CaM}$ kinase II activity becomes autonomous (the "on state"), autonomous kinase activity can persist after intracellular $\mathrm{Ca}^{2+}$ concentrations return to the basal level of less than $100 \mathrm{nM}$. This unique property of CaM kinase II leads to the hypothesis that autophosphorylation of the enzyme allows it to function as a memory molecule encoding frequency of synaptic usage $(5,6)$.

The function of CaM kinase II as a frequency detector of $\mathrm{Ca}^{2+}$ oscillations was demonstrated using the immobilization and superfusion system of the multimeric form of recombinant $\alpha$ - and $\beta$-CaM kinase II. The affinity for $\mathrm{CaM}$ of $\beta$-CaM kinase II is higher than that of $\alpha$ CaM-kinase II. The generation of autonomy is dependent on the frequency and duration of $\mathrm{Ca}^{2+}$ spikes as well as 
the free CaM concentration (7) (Fig. 2). For example, the $\mathrm{Ca}^{2+}$ spike at $4 \mathrm{~Hz}$ with 200 -msec duration maximally generates autonomous kinase activity with different concentrations of CaM. Interestingly, prephosphorylated CaM kinase II having $15 \% \mathrm{Ca}^{2+}$-independence can further potentiate its autonomous activity in response to a $1-\mathrm{Hz} \mathrm{Ca}^{2+}$-spike, which has no apparent effect on the unphosphorylated kinase. This is probably due to the functional cooperativity described above and suggests that the high autonomous kinase can maintain its autophosphorylated state at subthreshold activation (7). In support of this idea, LTP-inducing high frequency stimuli lead to an increase in the level of $\mathrm{Ca}^{2+}$-independent CaM kinase II activity in hippocampal slices (8). In hip- pocampal slices, the level of $\mathrm{Ca}^{2+}$-independence of $\mathrm{CaM}$ kinase II was about $12 \%$ in the basal condition and increased to about $14 \%$ following LTP-inducing stimulation. Increased autonomous kinase activity is relatively stable for at least $1 \mathrm{hr}$. Since stable LTP induction is also associated with an increase in total CaM kinase II activity, a substantial increase in $\mathrm{Ca}^{2+}$-independent activities in LTP-induced slices is more than $20 \%$ compared to that of control slices without high frequency stimuli. If the expression of LTP occurs in a limited dendritic spine, the increases in observed autonomous kinase activity seem to be large enough to produce long-lasting biochemical changes in critical synapses. The large extent of basal autonomous $\mathrm{CaM}$ kinase II activity $\left(8-15 \% \mathrm{Ca}^{2+}\right.$-in-
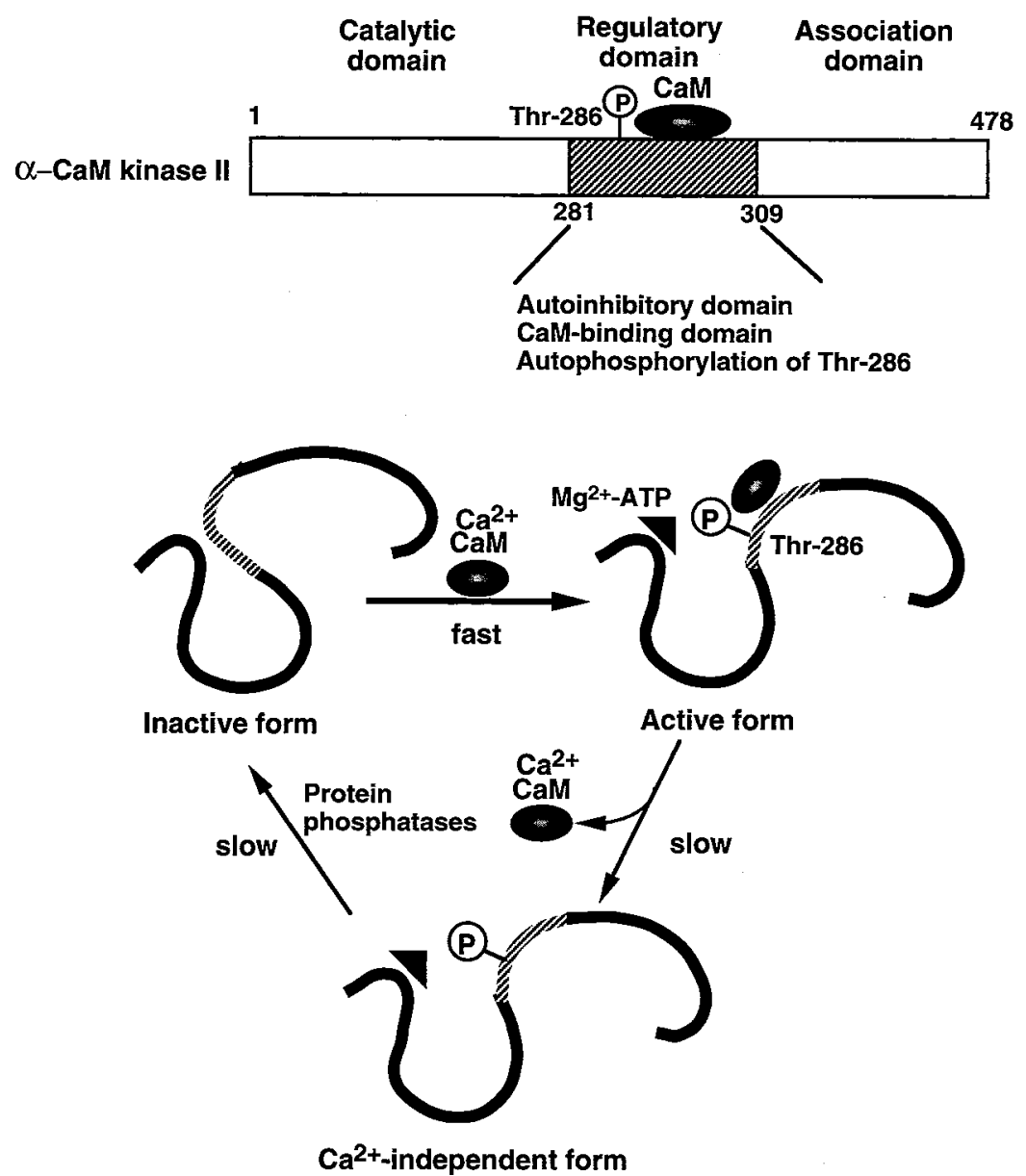

Fig. 1. Structure and activation mechanism of CaM kinase II. CaM kinase II is composed with the catalytic, regulatory and association domains. The regulatory domain of the enzyme that includes the CaM-binding site acts as an inhibitory domain by masking the catalytic domain including ATP- and substrate-binding sites. The binding of CaM to the binding site in the regulatory domain causes a conformational change of the enzyme and thereby removes the inhibitory action of the regulatory domain for the catalytic domain. Consequently, ATP can access to the catalytic domain and the enzyme is activated. When CaM kinase II is activated with $\mathrm{Ca}^{2+} / \mathrm{CaM}$, the site of $\mathrm{Thr}-286 / 287(\alpha / \beta$ subunit), which is located within the regulatory domain, is first autophosphorylated. Since the autophosphorylation disrupts the interaction between the autoinhibitory domain and the catalytic domain, CaM kinase II is active even in the absence of $\mathrm{Ca}^{2+} / \mathrm{CaM}$ and becomes a $\mathrm{Ca}^{2+}$-independent species

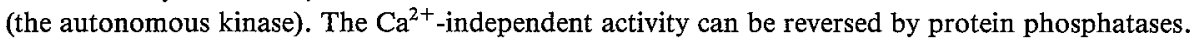




\section{LOW FREQUENCY STIMULATION}

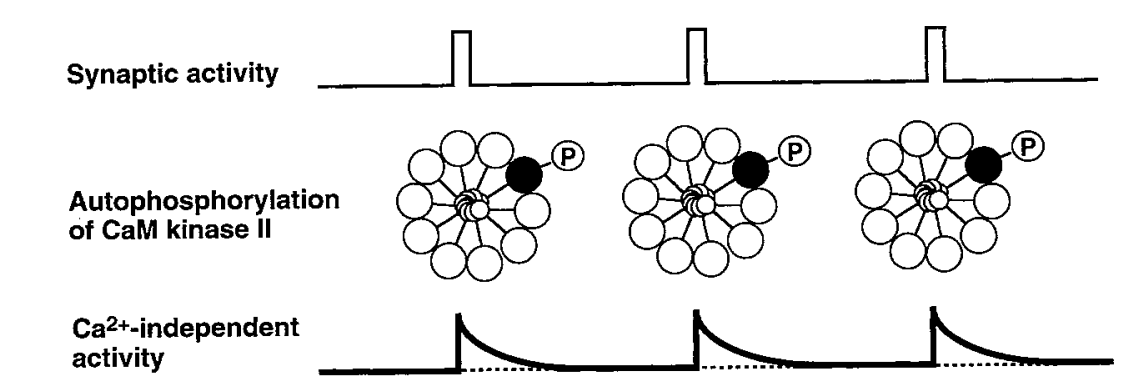

HIGH FREQUENCY STIMULATION

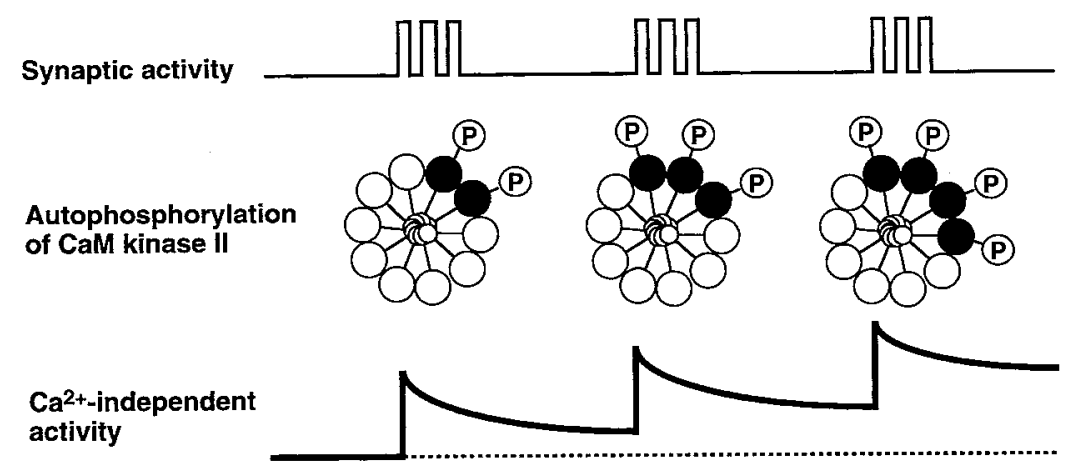

Fig. 2. Generation of the autonomous CaM kinase II activity by high frequency stimulation but not by low frequency stimulation. If the concentration of free $\mathrm{CaM}$ in the synaptic regions is stable, the generation of autonomy is dependent on the frequency and duration of $\mathrm{Ca}^{2+}$ spikes. The low frequency stimulation of the excitatory synapses causes transient relatively small $\mathrm{Ca}^{2+}$ spikes. The transient increases in the $\mathrm{Ca}^{2+}$ spikes fail to potentiate the autonomous kinase activity through the autophosphorylation. In contrast, the high frequency stimulation causes a large increase in the $\mathrm{Ca}^{2+}$ spikes in both pre- and postsynaptic regions. The accumulating $\mathrm{Ca}^{2+}$ spikes can promote the generation of the autonomous kinase activity as well as autophosphorylation of $\mathrm{Thr}-286$.

dependence) observed in adult rat hippocampus contrasts to that seen in immature neurons cultured from neonatal rat hippocampus (4-6\% $\mathrm{Ca}^{2+}$-independence) (9). Although stimulation with glutamate agonists or depolarization generates autonomous activity in cultured neurons, the increases are transient and persist only in the continued presence of agonists in the medium. Cultured neurons, which form a small number of excitatory synapses, cannot maintain the subthreshold state required to generate stable autophosphorylation and therefore exhibit low autonomous activity.

\section{Involvement of CaM kinase II autophosphorylation in LTP/LTD induction}

Knockout of the $\alpha$-CaM kinase II gene provides strong evidence for involvement of the enzyme in LTP as well as in spatial learning $(10,11)$. Also, infection of hippocampal slices with a vaccinia virus carrying a truncated, $\mathrm{Ca}^{2+}$-independent $\mathrm{CaM}$ kinase II gene potentiates synap- tic transmission and blocks LTP induction (12). To further test the role of CaM kinase II autophosphorylation in synaptic plasticity, Mayford et al. (13) conducted elegant studies with transgenic mice that express $\mathrm{CaM}$ kinase II-Asp-286 in the hippocampus. When Thr-286 is replaced with the acidic aspartate residue (CaM kinase IIAsp-286), which mimics autophosphorylation, the mutant kinase is 20 - to 30 -fold more active in the absence of $\mathrm{Ca}^{2+}$ than the wild-type enzyme. In this study, the CaM kinase II promoter was used to drive the mutant transgene and restrict its expression to the forebrain. The total $\mathrm{Ca}^{2+}$-independent activity increased from $7 \%$ to $14 \%$ in the mutant mice without changes in the total activity observed in adult hippocampus. The mutation would be expected to potentiate excitatory synaptic transmission and occlude LTP or undergo LTP more readily. However, synaptic transmission is not substantially altered, and the transgenic mice still exhibit a normal LTP in response to stimulation at $100 \mathrm{~Hz}$ and rather favor the 
production of long-term depression (LTD) in response to stimuli in the range of $1-10 \mathrm{~Hz}$. It is also unexpected that the size and direction of LTD produced following $5-\mathrm{Hz}$ stimulation are strongly correlated with the level of $\mathrm{Ca}^{2+}$-independent CaM kinase II activity. These findings are inconsistent with observations made in hippocampal slices transfected with a truncated form of CaM kinase II(1-290), in which the expression of LTP was occluded (12). Mayford et al. speculate that if the CaM-binding ability of CaM kinase II-Asp-286 mimics autophosphorylation, the mutant kinase may trap a large amount of $\mathrm{CaM}$ and result in a decrease in the concentration of free $\mathrm{Ca}^{2+} / \mathrm{CaM}$ required to activate other enzymes involved in LTP. This would in turn lead to a systematic shift in favor of LTD at submaximal levels of $\mathrm{Ca}^{2+}$ influx (i.e., at low frequency stimulation). In addition, the transgenic mice express the active kinase in both presynaptic and postsynaptic cells. This is in contrast to the postsynaptic expression of truncated CaM kinase II in neurons transfected with a vaccinia virus. Lack of CaM binding to the truncated form is also an important difference compared to CaM kinase II-Asp-286 mutants. Using the same strategy, Giese et al. (14) tried to determine whether autophosphorylation of Thr-286 is critical for LTP induction in the hippocampus. A point mutation of Thr-286 to Ala-286 was introduced into the $\alpha$-CaM kinase II gene, blocking autophosphorylation without affecting $\mathrm{Ca}^{2+}$ /CaM-dependent activity. They confirmed that the mutation decreased total $\mathrm{Ca}^{2+}$-independent activity in the mutants (about $9 \% \mathrm{Ca}^{2+}$-independent) compared to wild-type mice (about $22 \%$ ) without changing total kinase activity. Like CaM kinase II-Asp-286 mutants, the CaM kinase II-Ala-286 mutants still have normal synaptic transmission. However, the mutant mice had no NMDAreceptor-dependent LTP in the hippocampal CA1 region and showed deficits in spatial learning in the Morris water maze. Thus, the autophosphorylation of Thr-286 is a molecular switch underlying LTP and learning.

\section{Biochemical mechanisms of LTP/LTD induction}

Although CaM kinase II is established as a memory molecule encoding the frequency of synaptic usage, the molecular mechanisms underlying LTP and LTD are still unclear. Of course, the mechanisms of LTP/LTD are not uniform in different regions of the brain. For example, LTP induction of the Schaffer collateral/commissural synapses in CA1 regions of the hippocampus involves a postsynaptic mechanism and that of the mossy fiber synapses in the $\mathrm{CA} 3$ region is presynaptic (15). Here, we focus on the mechanism of LTP/LTD in the CA1 region. In the case of postsynaptic mechanisms, recent studies suggest that many synapses in the early postnatal hippocampus have only NMDA receptors and may not express functional AMPA receptors, thus making these synapses silent at the resting membrane potential. When these silent synapses are subjected to LTP-inducing stimuli, excitatory postsynaptic currents (EPSCs) mediated by AMPA receptors appear. This conversion of silent synapses to functional ones is an NMDA receptor-dependent process, which may explain the presynaptic changes in quantal content $(16,17)$. Activation of CaM kinase II may account for maturation of synapses from silent to functional. In the tadpole optic tectum, rostral cells infected postsynaptically with constitutively active CaM kinase II showed an increase in AMPA-receptor transmission with little change in NMDA-receptor transmission (18). Likewise, in CA1 pyramidal neurons, infusion of a constitutively active CaM kinase II via the recording pipette resulted in an increase in the size of EPSCs, as well as an increase in the amplitude of responses to iontophoretically applied AMPA (19). The enhancing action of CaM kinase II was greatly diminished by prior induction of LTP. Conversely, following the increase in synaptic strength mediated by CaM kinase II, tetanic stimulation failed to induce LTP. These findings lead to a simple switch model of LTP in which CaM kinase II alone is sufficient to induce LTP. However, mutant mice expressing the autonomous CaM kinase II both pre- and postsynaptically do not show significant potentiation in basal synaptic transmission, and they exhibit a normal LTP in response to stimulation of $100 \mathrm{~Hz}$ (13). In addition, at low frequencies in the range of $1-10 \mathrm{~Hz}$, there is a systematic shift in size and direction of the synaptic change to favor LTD in the CA1 region. The observations with the knockout mice of the $\alpha$-CaM kinase II gene also suggest that CaM kinase II has ability either to potentiate or to depress excitatory synaptic transmission depending on the pattern of presynaptic activation (20). Taken together, the activity of CaM kinase II in presynaptic sites could bidirectionally modulate the neurotransmitter release and may determine direction of either LTP or LTD.

Several investigations address how CaM kinase II selectively enhances AMPA responses and maintains functional synapses. In early-phase LTP, phosphorylation of the AMPA receptor by CaM kinase II may promote increased sensitivity in excitatory synaptic transmission. An increased phosphorylation of the type 1 glutamate receptor (GluR1) was associated with LTP induction and stable for $1 \mathrm{hr}$ following LTP, similar to the increased autophosphorylation of CaM kinase II (21). The phosphorylation of AMPA receptors by CaM kinase II results in an increase in the single-channel conductance of the receptor-ion channel (21). In late-phase LTP, the maintenance of increased AMPA responses seems to require expression of newly synthesized AMPA receptors, a process in which the cAMP-dependent protein kinase 
is involved (22). In addition to the AMPA receptor, increased phosphorylation of microtubule-associated protein 2 (MAP2) and synapsin I by CaM kinase II is evident in CA1 LTP (23). Interestingly, LTP induction was also associated with decreased protein phosphatase $2 \mathrm{~A}$ activity, in which the regulatory subunit, B', was phosphorylated by CaM kinase II (24).

According to the simple switch model of LTP induction through CaM kinase II autophosphorylation, decreased autophosphorylation underlies LTD in CA1 hippocampus neurons. One form of LTD in the CA1 region requires activation of postsynaptic NMDA receptors and results in increases in postsynaptic $\mathrm{Ca}^{2+}$ concentration. The increased $\mathrm{Ca}^{2+}$ concentration, which may be too low to stimulate CaM kinase II, activates the $\mathrm{Ca}^{2+} / \mathrm{CaM}$-dependent protein phosphatase, calcineurin, and results in dephosphorylation of inhibitor 1 . The dephosphorylation of inhibitor 1 leads to increased protein phosphatase 1 activity which accounts for the decrease in autonomous CaM kinase II activity in the postsynaptic density. In support of this idea, bath application of FK506, a calcineurin inhibitor, or infusion of phosphorylated inhibitor 1 into postsynaptic cells prevents LTD induction (25). However, this complex cascade requiring dephosphorylation of CaM kinase II does not account for NMDA-dependent LTD in CA1 regions. Because $\alpha$-CaM kinase II knockout mice have impaired LTD induction as well as LTP (26) and mutant mice have high level of $\mathrm{Ca}^{2+}$-independent activity by expressing the constitutively active kinase rather favor the production of LTD as discussed above (13). In addition, increased autophosphorylation was still observed in the CA1 region following LTD induction (K. Fukunaga et al., unpublished observation). In this context, dephosphorylation by calcineurin of other target proteins such as the inositol-3-phosphate $\left(\mathrm{IP}_{3}\right)$ receptor and voltage-dependent potassium channels may underlie the postsynaptic mechanisms of LTD. In addition to NMDA-receptordependent LTD, metabotropic glutamate receptor (mGluR)-dependent LTD is evident in CA1 LTD. The mGluR-LTD requires activation of T-type $\mathrm{Ca}^{2+}$ channels, group $1 \mathrm{mGluRs}$ and protein kinase $\mathrm{C}$ (PKC). Activation of mGluR1 may lead to a decrease in neurotransmitter release from presynaptic sites since a large decrease in the frequency of EPSCs occurs without changes in quantal size, and LTD induced by synaptic activation still requires activation of NMDA receptors in postsynaptic regions, in which retrograde messengers such as arachidonic acid and/or platelet activating factor are released in an NMDA receptor-dependent manner. Furthermore, use-dependent increases in glutamate concentration in the extracellular space may promote activation of presynaptic metabotropic glutamate receptors as seen in hippocampal mossy fiber synapses (27). The increase in extracellular glutamate is due partly to prolonged stimulation of neurotransmitter release by low frequency stimulation and to a block in glutamate uptake by surrounding astrocytes.

As summarized in Fig. 3, the accumulated large increase in postsynaptic $\mathrm{Ca}^{2+}$ resulting from high frequency stimulation leads to generation of autonomous CaM kinase II and results in phosphorylation of the AMPA receptor in postsynaptic regions. The decreased protein phosphatase $2 \mathrm{~A}$ and protein phosphatase 1 activities through CaM kinase II (24) and cAMP-dependent protein kinase (PKA) (28), respectively, can maintain persistent increases in phosphorylation of synaptic elements including the AMPA receptor as well as autophosphorylation of CaM kinase II. Together with changes in protein phosphatase activities, newly synthesized CaM kinase II accounts for the long-lasting increase in autophosphorylation in the specific dendritic spine receiving high frequency input. In addition to CaM kinase II, PKC has regulatory roles on LTP expression in postsynaptic neurons: postsynaptic injection of $\mathrm{PKC}$ inhibitory peptides inhibits tetanus-induced LTP and mice lacking $\mathrm{PKC} \gamma$ have mild deficits in LTP induction in the CA1 region. The modulatory role of PKC is due partly to phosphorylation of neurogranin and GAP 43 in the post synaptic and presynaptic regions, respectively. Phosphorylation of $\mathrm{CaM}$ binding proteins leads to an increase in free $\mathrm{CaM}$ concentration in the cytoplasm and thereby potentiates CaM-dependent pathways.

In contrast, LTD or depotentiation of LTP can be produced by a long-lasting stimulation with low frequency $(900$ pulse at $1 \mathrm{~Hz})$. The submaximal repetitive elevation of $\mathrm{Ca}^{2+}$ in postsynaptic cells leads to long-lasting activation of calcineurin in postsynaptic regions and usedependent increases in the concentration of extracellular glutamate. The increased glutamate would facilitate activation of mGluR or presynaptic kainate receptors (29) in presynaptic sites and in turn inhibit glutamate release. The repetitive elevation of $\mathrm{Ca}^{2+}$ in presynaptic regions may also stimulate PKC and/or CaM kinase II. Although the precise mechanism underlying inhibition of glutamate release is not well understood, $\mathrm{N}$-type $\mathrm{Ca}^{2+}$ channels are attractive target proteins for $\mathrm{PKC}$ and $\mathrm{CaM}$ kinase II because phosphorylation of the N-type $\mathrm{Ca}^{2+}$ channel inhibits its interaction with SNARE complexes containing syntaxin and SNAP-25 (30).

\section{Maintenance and consolidation mechanisms of LTP}

In contrast to LTP induction, the mechanism of maintenance and consolidation is poorly understood. The late-phase of LTP is believed to require synthesis of new proteins, since LTP maintenance is associated with 

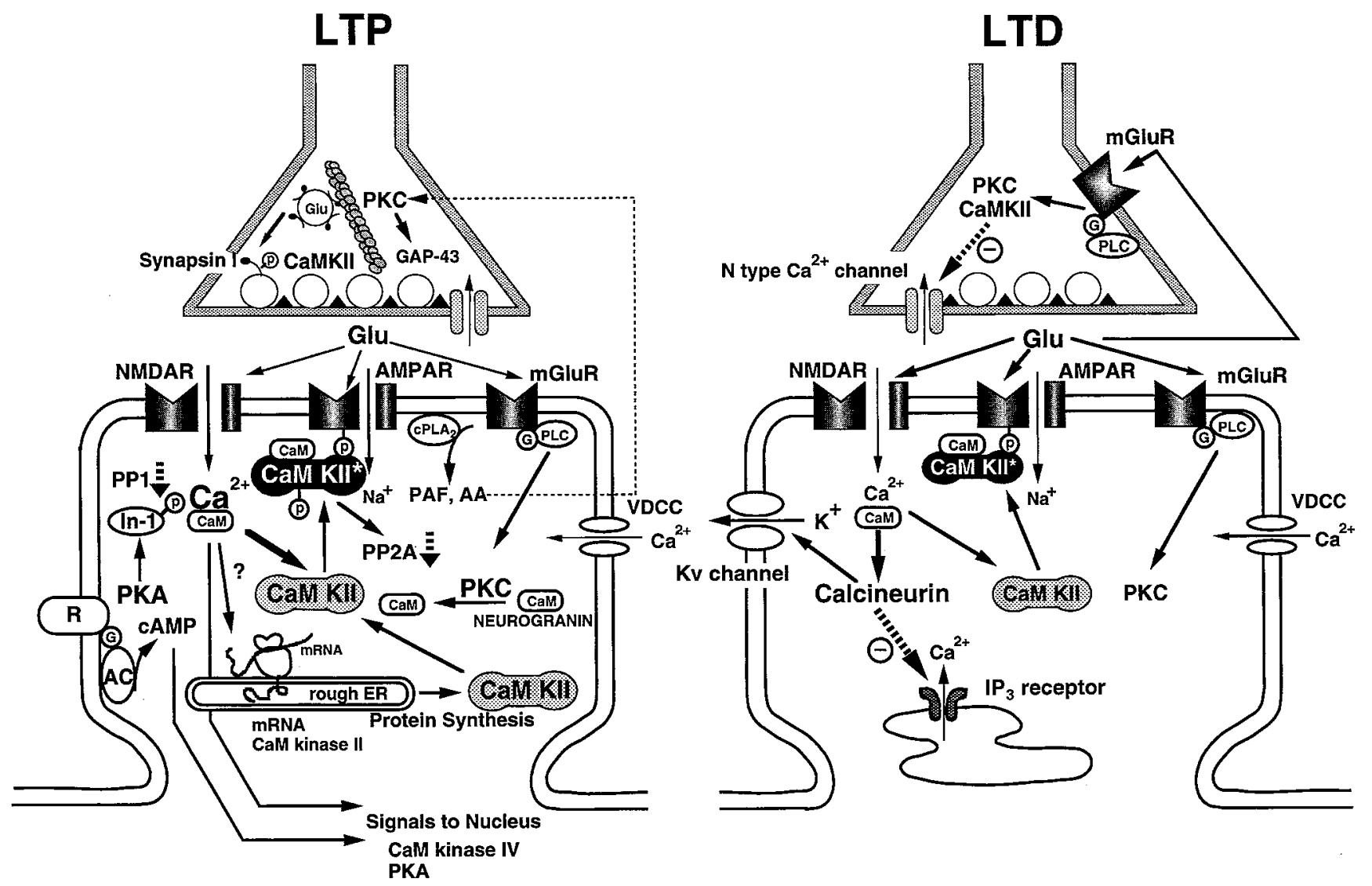

Fig. 3. Working hypothesis on the role of protein kinases in the induction of LTP and LTD. Tetanic stimulation of afferents in the CA1 region of the hippocampus induces glutamate release and activation of glutamate receptors in dendritic spines. A large increase in $\left[\mathrm{Ca}^{2+}\right]_{i}$, resulting from the influx through NMDA receptors, leads to generation of the $\mathrm{Ca}^{2+}$-independent form (CaM KII*) of CaM kinase II through autophosphorylation of Thr-286. Activation of the NMDA receptor and the metabotropic glutamate receptor ( $\mathrm{mGluR}$ ) are positively linked to activation of PKA through adenylate cyclase (AC) and activation of PKC, respectively. Inhibition of protein phosphatase 2A (PP2A) activity by CaM kinase II and of protein phosphatase 1 (PP1) by PKA through phosphorylation of inhibitor 1 (In-1) may maintain autonomous kinase activity. Both protein phosphatases dephosphorylate Thr-286 of the kinase. Newly synthesized CaM kinase II translated from preexisting mRNA may account for an increase in total CaM kinase II activity. Taken together, the long-lasting enhancement of CaM kinase II activity increases the sensitivity of AMPA receptors in postsynaptic sites. Stimulation of mGluRs leads to stimulation of PKC activity. The increased phosphorylation of neurogranin and GAP 43 by PKC in the postsynaptic and presynaptic sites, respectively, could increase free $\mathrm{CaM}$ concentrations and thereby potentiate CaM-dependent signaling, including CaM kinase II in both sites. Finally, large increases in $\mathrm{Ca}^{2+}$ and cAMP in turn activate CaM kinase IV and PKA to stimulate gene expression in the nucleus. In contrast, prolonged stimulation with low frequency causes LTD or depotentiation of established LTP. Low frequency and repetitive stimulation of postsynaptic glutamate receptors produces a submaximal increase in $\mathrm{Ca}^{2+}$ through NMDA and mGluRs. The submaximal increase in $\left[\mathrm{Ca}^{2 \dagger}\right]_{\mathrm{i}}$ in postsynaptic sites effectively stimulates calcineurin activity. Although there is no direct evidence in hippocampal LTD, the $\mathrm{IP}_{3}$ receptor and voltage-dependent $\mathrm{K}^{+}$channels but not CaM kinase II are potential targets for calcineurin. In addition, increased glutamate concentrations in the extracellular space produced by repetitive stimulation may stimulate mGluRs in presynaptic nerve terminals and thereby stimulate PKC and/or CaM kinase II. The N-type $\mathrm{Ca}^{2+}$ channel is an attractive candidate for $\mathrm{PKC}$ and $\mathrm{CaM}$ kinase II, since in vitro phosphorylation by both kinases inhibits the interaction between $\mathrm{N}$-type $\mathrm{Ca}^{2+}$ channels and SNARE proteins, which comprise a secretory machinery.

sprouting of synapses and/or morphological changes in dendritic spines. Evidence using protein synthesis inhibitors revealed that protein synthesis is necessary for maintenance and consolidation of LTP, in which proteins are synthesized from preexisting rather than newly transcribed mRNA. Since the existence of mRNAs encoding $\alpha$-CaM kinase II and MAP2 in neuronal dendrites has been demonstrated in hippocampal CA1 regions or cul- tured neurons, increased levels of $\alpha$-CaM kinase II and MAP2 proteins would be predicted. However, following LTP induction, significant increases were observed in $\beta$-CaM kinase II protein, and $\beta$-CaM kinase II mRNA is predominantly localized in the cell body (23). Levels of MAP2 protein actually decreased with LTP induction (23). Increased protein levels of CaM kinase II is also confirmed by immunohistochemistry (31). These changes 
in total CaM kinase II protein were observed within $1 \mathrm{hr}$ of tetanization. Anisomycin, an inhibitor of translation, was injected immediately after tetanization and produced a decay in LTP. When injected after a 15 min delay, the drug had no effect. Taken together, the early protein synthesis required for LTP maintenance is mostly complete within 15 min of tetanization. In addition, Machler et al. (32) observed a threefold increase in the CaM kinase II mRNA level between $30 \mathrm{~min}$ and $3 \mathrm{hr}$ after tetanization. Similarly, in the dentate gyrus of freely moving rats, transient expression of $\alpha$-CaM kinase II mRNA was observed in the soma, and a more persistent increase in the $\alpha$-isoform mRNA was observed in the dendritic field without changes in $\beta$-isoform mRNA following LTP induction (33). In addition, the mRNA levels of $\mathrm{PKC}$, extracellular signal-regulated kinase 2 (ERK2) and Raf B kinase transiently increased following induction of LTP in the dentate gyrus. Persistent activation of kinases has been thought to be required only in the early phase of LTP induction, since inhibitors of CaM kinase II and PKC do not eliminate established LTP if they are applied in the maintenance phase. However, Nayak et al. (22) found that synthesis of AMPA-receptor subunits increased $3 \mathrm{hr}$ after LTP induction in CA1 regions in which the cell bodies of presynaptic Schaffer collateral/commissural fibers are removed. The effect on synthesis of AMPA receptors is blocked by inhibitors of PKA and inhibitors of transcription. Thus, PKA may play critical roles in LTP maintenance as described previously (34). Induction of AMPA receptors through activation of PKA may be mediated by the CREB family of transcription factors: mice lacking the $\alpha$ - and $\delta$-isoforms of CREB are deficient in long-term memory and the maintenance phase of LTP. In addition to the CAMP pathway, $\mathrm{Ca}^{2+}$ waves from dendritic spines to the nucleus possibly account for regulation of LTP-induced transcription (35). However, which $\mathrm{Ca}^{2+}$-dependent protein kinases are involved in nuclear events have not been identified.

In LTP maintenance, newly synthesized CaM kinase II would account for stabilization of usage-dependent synaptic plasticity in specified dendrites that have received tetanic stimulation. How translation of the preexisting CaM kinase II mRNA is regulated is not clear, although the regulation occurs within 15-30 min. Following local up-regulation of translation of CaM kinase II, cAMP and/or $\mathrm{Ca}^{2+}$ signals can trigger transcription of synaptic elements, including AMPA receptors. Induction of mRNAs associated with LTP is observed $30 \mathrm{~min}$ to $3 \mathrm{hr}$ after tetanic stimulation. Indeed, a significant increase in the AMPA receptor protein is evident $3 \mathrm{hr}$ after LTP induction. If phosphorylation of the AMPA receptor is required for insertion into synaptic membranes, only dendritic spines having active CaM kinase II could express functional AMPA receptors. Further investigation is required to identify LTP-associated proteins expressed both pre- and postsynaptically. LTP maintenance is associated with morphological changes in dendritic spines, in which presynaptic changes in structure should be coordinated with postsynaptic events.

\section{Conclusion and questions for the future}

In the past decade, elegant molecular biological studies using transgenic mice have analyzed the physiological basis of LTP involved in learning and memory. Mice lacking $\alpha$-CaM kinase II are deficient in spatial learning as well as in the induction phase of LTP (11). New molecular technologies have achieved both regional and temporal control of transgene expression by combining a forebrain-specific promoter with the tetracycline transactivator system. Using this system, Mayford et al. demonstrated that the CaM kinase II signaling pathway is critical for both explicit and implicit memory (36). One form of implicit memory, fear conditioning, which is an amygdala-dependent form of learning associated with LTP (37), is impaired in CaM kinase II mutant mice having autonomous kinase activity (36). Furthermore, mutant mice expressing $\alpha$-CaM kinase II-Ala-286 or lacking $\alpha$ and $\delta$-isoforms of CREB showed abnormal representation of place cells in the hippocampus during spatial learning (38). Thus, hippocampal LTP is important not only for hippocampal-dependent spatial memory but also for amygdala-dependent implicit memory. In both cases, autophosphorylation of $\mathrm{CaM}$ kinase II may encode the frequency of synaptic usage and maintain synaptic facilitation.

In addition to synaptic plasticity in pyramidal neurons, a novel form of synaptic plasticity has been observed in hippocampal GABAergic interneurons (39). This finding is potentially important since GABAergic interneurons spontaneously repeat firing and generate subthreshold oscillations in hippocampal pyramidal neurons at $\theta$ frequency $(4-7 \mathrm{~Hz})$, which is associated with exploration in rodents. Indeed, patterned stimulation in the range of 5 $\mathrm{Hz}$ is especially effective in producing LTP. Disruption of the $\theta$ rhythm by lesions in cholinergic inputs to the hippocampus impairs spatial memory. CaM kinase II mutant mice have impairment of spatial learning as well as selective loss of CA1 LTP in the range of the $\theta$ frequency. Thus, the oscillation of GABAergic interneurons play a critical role in determining the direction between LTP and LTD. To clarify the molecular basis of maintenance and consolidation of synaptic plasticity underlying learning and memory, it is now essential to understand both the role of the $\theta$ rhythm in spatial memory and the GABAergic control of hippocampal synaptic plasticity through the $\theta$ rhythm. Transgenic mice will continue to provide tools required for these studies during the next decade. 


\section{REFERENCES}

1 Fukunaga $\mathrm{K}$, Muller D and Miyamoto E: CaM kinase II in long-term potentiation. Neurochem Int 28, 343-358 (1996)

2 Miyamoto $\mathrm{E}$ and Fukunaga $\mathrm{K}$ : A role of $\mathrm{Ca}^{2+} /$ calmodulindependent protein kinase II in the induction of long-term potentiation in hippocampal CA1 area. Neurosci Res 24, 117-122 (1996)

3 Hanson PI, Meyer T, Stryer L and Schulman H: Dual role of calmodulin in autophosphorylation of multifunctional CaM kinase may underlie decoding of calcium signals. Neuron $\mathbf{1 2}$, 943-956 (1994)

4 Meyer T, Hanson PI, Stryer L and Schulman H: Calmodulin trapping by calcium-calmodulin-dependent protein kinase. Science 256, 1199-1202 (1992)

5 Lisman JA: Mechanism for the Hebb and anti-Hebb processes underlying learning and memory. Proc Natl Acad Sci USA 86, 9574-9587 (1989)

6 Miller SG and Kennedy MB: Regulation of brain type II Ca ${ }^{2+}$ /calmodulin-dependent protein kinase by autophosphorylation: $\mathrm{A} \mathrm{Ca}^{2+}$-triggered molecular switch. Cell 44, 861-870 (1986)

7 De Koninck P and Schulman H: Sensitivity of CaM kinase II to the frequency of $\mathrm{Ca}^{2+}$ oscillations. Science 279, 227-230 (1998)

8 Fukunaga K, Stoppini L, Miyamoto E and Muller D: Longterm potentiation is associated with an increased activity of $\mathrm{Ca}^{2+} /$ calmodulin-dependent protein kinase II. J Biol Chem 268, $7863-7867$ (1993)

9 Fukunaga K, Soderling TR and Miyamoto E: Activation of $\mathrm{Ca}^{2+} /$ calmodulin-dependent protein kinase II and protein kinase $\mathrm{C}$ by glutamate in cultured rat hippocampal neurons. J Biol Chem 267, 22527-22533 (1992)

10 Silva AJ, Stevens CF, Tonegawa S and Wang Y: Deficient hippocampal long-term potentiation in alpha-calcium-calmodulin kinase II mutant mice. Science 257, 201-206 (1992)

11 Silva AJ, Paylor R, Wehner JM and Tonegawa S: Impaired spatial learning in alpha-calcium-calmodulin kinase II mutant mice. Science 257, 206-211 (1992)

12 Pettit DL, Perlman S and Malinow R: Potentiated transmission and prevention of further LTP by increased CaMKII activity in postsynaptic hippocampal slice neurons. Science 266, 1881 - 1885 (1994)

13 Mayford M, Wang J, Kandel ER and O'Dell TJ: CaMKII regulates the frequency-response function of hippocampal synapses for the production of both LTD and LTP. Cell 81, 891-904 (1995)

14 Giese KP, Fedorov N, Filipkowski RK and Silva AJ: Autophosphorylation at Thr286 of the alpha calcium-calmodulin kinase II in LTP and learning. Science 279, 870-873 (1998)

15 Nicoll RA and Malenka RC: Contrasting properties of two forms of long-term potentiation in the hippocampus. Nature 377, 115-118 (1995)

16 Liao D, Hessler NA and Malinow R: Activation of postsynaptically silent synapses during pairing-induced LTP in CA1 region of hippocampal slice. Nature 375, 400-404 (1995)

17 Isaac JT, Nicoll RA and Malenka RC: Evidence for silent synapses: implications for the expression of LTP. Neuron 15, 427-434 (1995)

18 Wu G, Malinow R and Cline HT: Maturation of a central glutamatergic synapse. Science 274, 972-976 (1996)
19 Lledo PM, Hjelmstad GO, Mukherji S, Soderling TR, Malenka RC and Nicoll RA: Calcium/calmodulin-dependent kinase II and long-term potentiation enhance synaptic transmission by the same mechanism. Proc Natl Acad Sci USA 92, 1117511179 (1995)

20 Chapman PF, Frenguelli BG, Smith A, Chen CM and Silva AJ: The alpha- $\mathrm{Ca}^{2+} /$ calmodulin kinase II: a bidirectional modulator of presynaptic plasticity. Neuron 14, 591-597 (1995)

21 Barria A, Muller D, Derkach V, Griffith LC and Soderling TR: Regulatory phosphorylation of AMPA-type glutamate receptors by CaM-KII during long-term potentiation. Science 276, 2042-2045 (1997)

22 Nayak A, Zastrow DJ, Lickteig R, Zahniser NR and Browning MD: Maintenance of late-phase LTP is accompanied by PKAdependent increases in AMPA receptor synthesis. Nature 394, 680-683 (1998)

23 Fukunaga $\mathrm{K}$, Muller $\mathrm{D}$ and Miyamoto E: Increased phosphorylation of $\mathrm{Ca}^{2+} /$ calmodulin-dependent protein kinase II and its endogenous substrates in the induction of long-term potentiation. J Biol Chem 270, 6119-6124 (1995)

24 Fukunaga K, Muller D and Miyamoto E: Role of protein phosphatases in hippocampal long-term potentiation. Abs Soc Neurosci 22, 331 (1996)

25 Mulkey RM, Endo S, Shirish S and Malenka RC: Involvement of calcineurin/inhibitor-1 phosphatase cascade in hippocampal long-term depression. Nature 369, 486-488 (1994)

26 Stevens CF, Tonegawa S and Wang Y: The role of calciumcalmodulin kinase II in three forms of synaptic plasticity. Curr Biol 4, 687-693 (1994)

27 Scanziani M, Salin PA, Vogt KE, Malenka RC and Nicoll RA: Use-dependent increases in glutamate concentration activate presynaptic metabotropic glutamate receptors. Nature $\mathbf{3 8 5}$, 630-634 (1997)

28 Blitzer RD, Connor JH, Brown GP, Wong T, Shenolikar $S$, Lyengar R and Landau EM: Gating of CaMKII by cAMPregulated protein phosphatase activity during LTP. Science 280, 1940-1943 (1998)

29 Chittajallu R, Vignes M, Dev KK, Barnes JM, Collingridge GL and Henley JM: Regulation of glutamate release by presynaptic kainate receptors in the hippocampus. Nature 379, 78-81 (1996)

30 Yokoyama CT, Sheng Z-H and Catterall WA: Phosphorylation of the synaptic protein interaction site on $\mathrm{N}$-type calcium channels inhibits interactions with SNARE proteins. J Neurosci 17, $6929-6938$ (1997)

31 Ouyang Y, Kantor D, Harris KM, Schuman EM and Kennedy MB: Visualization of the distribution of autophosphorylated calcium/calmodulin-dependent protein kinase II after tetanic stimulation in the CA1 area of the hippocampus. J Neurosci 17, 5416-5427 (1997)

32 Mackler SA, Brooks BP and Eberwine JH: Stimulus-induced coordinate changes in mRNA abundance in single postsynaptic hippocampal CA1 neurons. Neuron 9, 539-548 (1992)

33 Thomas KL, Laroche S, Errington ML, Bliss TVP and Hunt SP: Spatial and temporal changes in signal transduction pathways during LTP. Neuron 13, 737-745 (1994)

34 Frey U, Huang YY and Kandel ER: Effects of cAMP simulate a late stage of LTP in hippocampal CA1 neurons. Science 260, $1661-1664$ (1993)

35 Deisseroth K, Bito H and Tsien RW: Signaling from synapse to 
nucleus: postsynaptic CREB phosphorylation during multiple forms of hippocampal synaptic plasticity. Neuron 16, 89-101 (1996)

36 Mayford M, Bach ME, Huang YY, Wang L, Hawkins RD and Kandel ER: Control of memory formation through regulated expression of a CaMKII transgene. Science 274, 1678-1683 (1996)

37 McKernan MG and Shinnick GP: Fear conditioning induces a lasting potentiation of synaptic currents in vitro. Nature $\mathbf{3 9 0}$, $607-611$ (1997)

38 Cho YH, Giese KP, Tanila H, Silva AJ and Eichenbaum $\mathrm{H}$ : Abnormal hippocampal spatial representations in $\alpha \mathrm{CaMKII}^{\mathrm{T} 286 \mathrm{~A}}$ and $\mathrm{CREB}^{\alpha \Delta-}$ mice. Science 279, 867-869 (1998)

39 McMahon LL and Kauer JA: Hippocampal interneurons express a novel form of synaptic plasticity. Neuron 18, 295-305 (1997) 